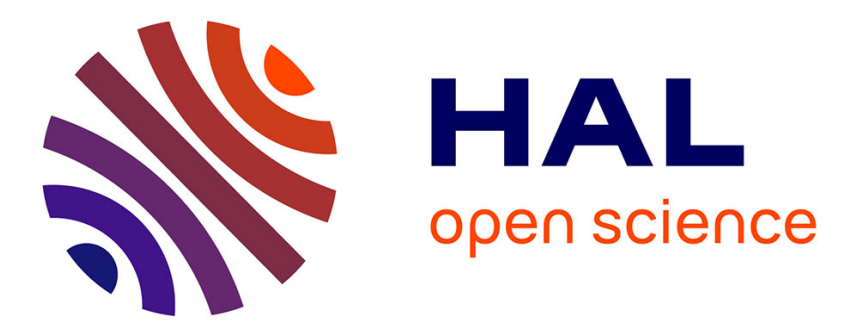

\title{
Les constructions causatives avec mouvement en allemand: d'une saisie phraséologique à une explication constructionnelle
}

\author{
Martine Dalmas, Laurent Gautier
}

\section{- To cite this version:}

Martine Dalmas, Laurent Gautier. Les constructions causatives avec mouvement en allemand: d'une saisie phraséologique à une explication constructionnelle. Langages, 2013, 189, pp.81-102. hal00812713

\section{HAL Id: hal-00812713 \\ https://u-bourgogne.hal.science/hal-00812713}

Submitted on 22 Feb 2016

HAL is a multi-disciplinary open access archive for the deposit and dissemination of scientific research documents, whether they are published or not. The documents may come from teaching and research institutions in France or abroad, or from public or private research centers.
L'archive ouverte pluridisciplinaire HAL, est destinée au dépôt et à la diffusion de documents scientifiques de niveau recherche, publiés ou non, émanant des établissements d'enseignement et de recherche français ou étrangers, des laboratoires publics ou privés. 
Martine Dalmas

Université Paris-Sorbonne \& Groupe de recherche 'Contextes, Variation, Usages'

(CoVariUs - UMS 3323)

Laurent Gautier

Université de Bourgogne \& Centre Interlangues Texte Image Langage (EA 4182)

\section{Les constructions causatives avec mouvement en allemand : d'une saisie phraséologique à une explication constructionnelle}

\section{PROBLÉMATIQUE}

La présente contribution cherche à répondre à la question posée par ce volume de Langages en s'arrêtant sur certaines structures causatives de l'allemand qui semblent particulièrement révélatrices de l'extension postulée ici du domaine de la phraséologie, extension concrètement envisagée à travers le développement récent des grammaires de construction, mais qui soulève le problème des critères de délimitation des deux modes $\mathrm{d}$ 'interprétation. L'interprétation constructionnelle, en particulier dans le sillage de A. Goldberg $(1995,2006)$, permet en effet, à la suite de H. Feilke (2007), d'envisager sous un jour nouveau maints phénomènes traditionnellement considérés comme relevant, au sens large, du préfabriqué langagier.

La démonstration qui va suivre trouve son point de départ dans la recherche phraséologique allemande telle qu'elle s'est développée depuis les années 80 pour finir par se constituer en discipline plus ou moins autonome à l'intérieur du champ des sciences du langage ${ }^{1}$. Si la recherche française sur des objets similaires s'est surtout articulée autour de la notion de figement

\footnotetext{
1. Les études réunies par Granger \& Meunier (2008) montrent bien, par contraste, que le « fait phraséologique » peut par ailleurs être abordé en dehors cette discipline pour devenir une problématique touchant à la description générale des langues naturelles. C'est en particulier le sens de la démonstration de Gries (2008) qui sera convoquée à plusieurs reprises dans les pages à venir et qui illustre, à nos yeux, une des instanciations de cette « extension de la phraséologie ».
} 
(Mejri 1997) et que la recherche anglo-saxonne a placé au centre de ses préoccupations l'aspect routinier et formulatoire de telles séquences (formulaic language, cf. Wray 2002, 2008), la tradition allemande s'est, de son côté, focalisée à la fois sur la reconnaissance de traits définitoires généraux réunis dans la triade polylexicalité/fixité/figuration ${ }^{2}$ (Fleischer 1982 ; Gréciano 1983 repris dans Burger 1998) et sur la mise au point de typologies, souvent multicritères, permettant de classer le matériau ainsi délimité. De ce point de vue, les structures causatives traitées ici peuvent être rapprochées, dans une première approximation, d'une des classes ainsi obtenues, celle des Funktionsverbgefüge ${ }^{3}$ (par la suite FVG) du type in Kraft setzen ('mettre en vigueur') :

(1) Oktober 1996 wurde das Gesetz in Kraft gesetzt.

'En octobre 1996, la loi a été mise en vigueur.' ${ }^{4}$

où le trait [+causatif] est lié, par rapport à la variante non causative en (2), à la substitution du verbe setzen au verbe treten:

(2) Januar 1990 ist das Gesetz in Kraft getreten.

'En janvier 1990, la loi est entrée en vigueur.'

Cette classe étant bien établie dans tout inventaire phraséologique de l'allemand, la question de l'extension du domaine de la phraséologie se pose en des termes singuliers : nous défendrons ici l'hypothèse que, par delà les structures totalement figées et largement étudiées ${ }^{5}$ du type de in Kraft treten/setzen en (1) et (2), un certain nombre d'autres phénomènes courants en allemand et servant directement l'expression de la causativité ne peuvent que bénéficier d'une approche constructionnelle, libérant l'analyse du carcan lexical (figement de tel $\mathrm{N}$ avec tel V) pour mettre au jour une structure sous-jacente à l'interface entre syntaxe et sémantique ${ }^{6}$ et permettant non seulement de décoder mais aussi d'encoder des énoncés à première vue peu figés comme en (3), voire " exotiques » comme en (4) :

\author{
Sie sang das Kind in den Schlaf. \\ 'Elle chantait pour endormir l'enfant.'
}

2. Voir la contribution de Schmale (2013), ce volume.

3. Pour des raisons de clarté terminologique et méthodologique, nous choisissons ici de ne pas traduire le terme allemand et, surtout, de ne pas recourir au terme français locution à verbe support dans la mesure où, contrairement à van Pottelberge (2001, 2007), nous défendons l'idée de la non-comparabilité des deux phénomènes du point de vue des traditions de recherche sous-jacentes (Gautier 2012, sous presse).

4. Pour la traduction de tous les exemples, nous avons renoncé à « l'élégance » pour privilégier une traduction proche de l'allemand afin de permettre aux lecteurs non-germanophones de comprendre les processus linguistiques à l'œuvre.

5. Cf. la monographie de van Pottelberghe (2001) et son imposante bibliographie.

6. Dans sa discussion des rapports entre phraséologie et théories linguistiques, Gries $(2008: 8)$ propose d'introduire une différenciation entre ce type d'interface, situé à un niveau très général, et une interface syntaxe-lexique permettant de poser comme critère de reconnaissance du phrasème la présence obligatoire d'au moins un élément lexical « contraint ». Nous reviendrons sur cet aspect dans le $\$ 4.2$ dans la mesure où se pose alors la question du degré de parenté des structures argumentales abstraites étudiées dans cet article avec la phraséologie ainsi définie. 
Heute hat mich einer von e-plus um 7 Uhr aus dem Bett geklingelt.

'Aujourd'hui, un type de e-plus m'a tiré du lit en me téléphonant à 7 heures.'

L'objet de la présente étude est donc constitué par une partie des structures rangées sous l'étiquette « résultatives » et analysées en détails pour l'anglais par J. Carrier et J. Randall (1992), puis B. Levin et M. Rappaport Hovav (1995, 2001), et que A. Goldberg (1995 : 152-179) a identifiées en anglais sous la dénomination de « constructions causatives avec mouvement » (caused-motion constructions). Deux raisons, intimement liées, motivent notre choix : la première tient à leur complexité syntactico-sémantique - surtout dans une langue à flexion casuelle comme l'allemand - favorisant un traitement au sein du paradigme constructionnel et la seconde à leur productivité, aspect qui les rapproche précisément du fait phraséologique dans la mesure où la mise en œuvre du schéma abstrait postulé ici, y compris pour les cas les moins prévisibles, laisse supposer que ce schéma est stocké comme un tout chez le locuteur. Nous suivons donc en cela H. Feilke (2007 : 63-64) lorsqu'il plaide pour aborder cette problématique dans le cadre plus général des rapports entre phraséologie et théorie générale de la langue : la reconnaissance de modèles syntactico-sémantiques relativement abstraits - que ceux-ci soient des constructions ou autre chose - recentre tout d'abord la recherche sur la question du continuum lexique/grammaire, là où la phraséologie de tradition allemande se situe plutôt du côté du seul lexique ${ }^{7}$ en traitant par exemple les défauts transformationnels comme des anomalies grammaticales ${ }^{8}$. Elle représente ensuite bel et bien une extension du domaine initialement associé au fait phraséologique en mettant au premier plan la dimension matricielle de ces structures qui devraient figurer dans l'inventaire des constructions de l'allemand ${ }^{9}$.

Pour répondre à ces questions, il conviendra de commencer par s'arrêter sur les traitements traditionnels de la causativité en allemand afin, d'une part, de montrer l'existence d'une structure syntactico-sémantique de base, candidate au statut de « construction » au sens technique du terme, et, d'autre part, de mieux comprendre le pourquoi des approches phraséologiques usuelles (section 2). Ce point de départ nous conduira à montrer ensuite la productivité de cette structure en allemand contemporain, y compris dans des contextes inattendus (verbes réputés intransitifs ou n'encodant à première vue ni déplacement ni mouvement), permettant ainsi de discuter les questions du complément directionnel et du constituant à l'accusatif (section 3). Au terme de cette discussion, la dernière partie se focalisera sur l'analyse sémantique de la construction en reposant la question esquissée ci-dessus du rapport à la phraséologie (section 4).

7. Voir note 6 supra.

8. Ceci est particulièrement le cas pour les FVG dont il est question dans les exemples 1 et 2 ; voir Helbig (1979).

9. Sur ce point, le rapprochement opéré par Feilke (2007: 66) entre constructions et schémas phrastiques (Satzbaupläne) d'inspiration valencielle dans la grammaire Duden est particulièrement parlant. 


\section{LA CAUSATIVITÉ EN ALLEMAND : APPROCHES TRADITIONNELLES}

\subsection{Approches définitoires}

La confrontation des définitions de la notion de causativité tant en français qu'en allemand permet de se faire une première idée de la façon dont cette catégorie est traitée dans les approches traditionnelles et « grammaticales ». Pour ce faire, ce sont les définitions de F. Neveu (2004:60-61) et du système d'information grammaticale en ligne grammis ${ }^{10}$ qui ont été retenues pour la comparaison. De celles-ci, il appert :

- que la notion opère, de prime abord, au niveau valenciel en modifiant, le cas échéant, la structure argumentale du verbe concerné. Cette modification peut alors être caractérisée, en première approximation, comme l'ajout d'un argument : ce type d'approche s'inscrit clairement dans un paradigme de recherche faisant reposer la structure argumentale de l'énoncé - et donc l'interprétation des relations entre les arguments - sur le sémantisme du seul verbe. Cet aspect sera particulièrement pertinent pour la suite de la démonstration dans la mesure où les structures causatives avec mouvement traitées ici peuvent «transformer » en verbes transitifs des verbes réputés intransitifs. Cette idée de transformation d'un programme valenciel verbo-centré a été largement discutée dans les études sur la résultativité ${ }^{11}$ et constitue, par ailleurs, l'une des brèches dans lesquelles s'engouffrent, pour mieux la réfuter, les grammaires de construction « à la Goldberg " ${ }^{12}$;

- que la notion doit ensuite aussi être abordée en termes de rôles sémantiques en ce qu'elle est directement liée à la notion d'agentivité : les prédicats mis en jeu ne peuvent être que des prédicats d'action avec une entité jouant le rôle d'agent volontaire et conscient déclenchant un certain type de mouvement, concret ou abstrait, pour une entité pouvant être appréhendée, en termes classiques, comme objet affecté ${ }^{13}$;

10. Ce système est géré par le Institut für deutsche Sprache de Mannheim (http://hypermedia.ids-mannheim.de/, consulté le 11.02.2012).

11. Notamment par Carrier \& Randall (1992 : 204), qui concluent : "The intransitive postverbal NP, then, must also be lexically governed by the verb, despite the fact that it is not an argument of that verb." Levin \& Rappaport Hovav (1995 : 48) les suivent et écrivent : "We will not take a stand on this issue, howewer, and will just stress that it is important for us that the postverbal NP is not an argument of the verb in resultative constructions based on unergative verbs, although it is in resultative constructions based on transitive verbs."

12. Voir Goldberg (1995: 11-12) : "The verb, on the other hand, is associated with one or a few basic senses which must be integrated into the meaning of the construction. [...] Instead of positing a new sense every time a new syntactic configuration is encountered and then using that sense to explain the existence of the syntactic configuration, a constructional approach requires that the issue of the interaction between verb meaning and constructional meaning must be addressed." (nous soulignons)

13. Selon une tradition bien ancrée en linguistique allemande, nous reprenons ici la terminologie et les concepts de von Polenz (1985). 
- que le fait d'associer au mouvement un lieu (but ou point de départ) modifie l'aspect (Aktionsart) du procès exprimé (activité > accomplissement) ${ }^{14}$;

- que cette notion de causativité, enfin, repose sur des réalisations langagières variées donnant une impression de peu de systématicité. Les exemples produits par grammis à l'appui de la définition appliquée au système de l'allemand recourent ainsi, d'une part, au verbe factitif lassen (5) et, d'autre part, à un verbe comme kochen en emploi transitif (6a) tandis que le même verbe $\mathrm{s}^{\prime}$ utilise aussi intransitivement (6b) sans modification morphologique particulière :

(5) Sie sollen aber in Zukunft besser auf sie achtgeben, lässt Ihnen der Herr Senator sagen...

'À l'avenir, vous devriez mieux faire attention à elle, vous fait transmettre le Sénateur...'

(6) a. Sie kocht weiße Rüben und einen Lammsbraten.

'Elle fait cuire des navets et un rôti d'agneau.'

b. Die Rüben kochen.

'Les navets cuisent.'

À y regarder de plus près, deux autre types de marquage méritent d'être examinés plus en détails : d'une part, parce qu'ils semblent offrir un début de systématicité et, d'autre part, parce qu'ils invitent au dépassement du « tout phraséologique » pour une approche plus analytique où les constructions trouvent toute leur place.

\subsection{Un mini-système morphologique}

Il existe tout d'abord un embryon de systématique au niveau du lexique à travers la dérivation morphologique possible de certains étymons qui connaissent deux formes : l'une non causative et l'autre causative à l'instar de la paire trinken/tränken dans les exemples (7) :

(7) a. Hallo, ich brauche mal ein paar Tips von euch, mein Pferd trinkt fast gar nichts, obwohl es so warm ist.

'Salut, j'ai besoin que vous me donniez quelques conseils, mon cheval ne boit presque rien bien qu'il fasse si chaud.'

b. Ein dritter Reiter kommt und tränkt das Pferd.

'Arrive un troisième cavalier, qui fait boire le cheval.'

Le raisonnement induit par les deux définitions discutées supra, à savoir celui de la modification de la structure argumentale sous-jacente, s'applique pleinement ici. Même si les deux énoncés comptent bien chacun deux actants, ils se différencient par les rôles sémantiques impliqués et la distribution du GN de base Pferd: tandis que l'énoncé (7a) présente mein Pferd en fonction de sujet et d'agent, et l'indéfini nichts en fonction d'objet (facultatif) à l'accusatif, l'énoncé 
(7b) s'ouvre sur un sujet présentant le trait |+HUMAIN $\mid$ et le GN de base Pferd, ici objet obligatoire, y apparaît comme patient de l'action.

Il en va exactement de même au sein du second mini-système traditionnellement illustré par la série des verbes dits « de position » et « de mouvement » et qui comprend les quatre paires sitzen/setzen (position assise), liegen/legen (position horizontale), stehen/stellen (position verticale) et hängen/hängen (position suspendue). Cette série est particulièrement intéressante car, compte de tenu de son ancrage initialement spatial ${ }^{15}$, elle contient en germe le " programme » mis en œuvre pour les constructions plus complexes qui sont au centre de notre analyse :

(8) a. Ein Kind liegt auf dem Bett und liest in einem Buch. 'Un enfant est allongé sur le lit et lit un livre.'

b. In aller Eile schließst Kari die Tür, legt das Kind auf das Bett und macht Feuer.

'En toute hâte, Kari ferme la porte, allonge l'enfant sur le lit et fait du feu.'

Au niveau morpho-syntaxique, un passage s'opère d'une structure à deux arguments en (8a) - GN au nominatif en fonction de sujet (ein Kind) et GPREP complément de lieu au datif (auf dem Bett), à une structure à trois arguments en ( $8 \mathrm{~b})$ : un GN au nominatif en fonction de sujet (Kari), un GN à l'accusatif en fonction d'objet (das Kind, qui apparaissait comme sujet grammatical de la variante noncausative) et un GPREP complément directif à l'accusatif (auf das Bett). En termes de rôles sémantiques, la structure non causative en (8a) comprend un expérienceur ${ }^{16}$ et un locatif tandis que la structure causative $(8 \mathrm{~b})$ comprend un agent, un patient et un directif, le patient correspondant à l'expérienceur de la structure précédente. C'est précisément cette structuration syntactico-sémantique minimale qui va donner lieu à des variations plus ou moins attendues, et non directement explicables à partir du seul verbe, en particulier lorsque celui-ci est sans lien direct avec le domaine du déplacement.

Avant d'en arriver là, il convient toutefois de s'arrêter sur un autre minisystème qui a donné lieu à une abondante littérature et à l'inscription de cette problématique dans le champ phraséologique, les Funktionsverbgefüge.

\subsection{La " récupération " phraséologique de la causativité}

Les Funktionsverbgefüge, littéralement « structures à verbe fonctionnel » ${ }^{17}$, sont des unités préfabriquées et relativement bien lexicographiées se présentant en surface sous deux formes:

15. «Initialement » spatial, car ces verbes connaissent tous des emplois métaphoriques.

16. Neveu (2004 : 257) propose deux traductions pour ce rôle sémantique dénommé experiencer en anglais : expérienceur et expérient.

17. Voir note 3 supra. 


$$
\begin{aligned}
& \text { préposition + marque casuelle / nom prédicatif / verbe fonctionnel } \\
& \text { zum Ausdruck kommen ('exprimer') } \\
& \text { nom prédicatif à l'accusatif / verbe fonctionnel } \\
& \text { Anzeige erstatten ('porter plainte') }
\end{aligned}
$$

Ce caractère préfabriqué et souvent sériel (cf. infra) ainsi que le comportement de ces structures par rapport aux critères définitoires mentionnés précédemment elles sont par définition polylexicales, relativement figées et leur sens n'est que faiblement compositionnel compte tenu du poids sémantique relativement faible du verbe fonctionnel - ont facilité leur intégration dans le domaine de la phraséologie ${ }^{18}$. Par delà la forme, ce sont les «fonctions » attribuées à ces structures qui retiennent l'attention pour notre démonstration. Depuis les travaux fondateurs de P. von Polenz (1963) et G. Helbig (1979), la recherche leur reconnaît quasi unanimement un rôle dans le marquage aspectuel comme en (11) où la variation sur les verbes kommen et bleiben permet de marquer l'inchoativité (11a) et la durativité (11b) :

(11) a. Wenn Proben noch am selben Tag zur Bearbeitung kommen, sind Lagerung und Transport bei Raumtemperatur immer ausreichend.

'Si les échantillons sont traités le jour même, il suffit de les stocker et de les transporter à température ambiante.'

b. Das Forum wird immer in Bearbeitung bleiben !! Is doch klar... das wird nie ganz fertig sein... da es immer neue Ideen gibt!!

'Le forum restera toujours en activité !! C'est évident... ce ne sera jamais fini... vu qu'il y a toujours de nouvelles idées !!'

L'autre apport de ces constructions est à chercher précisément du côté du marquage de la causativité, comme en (12) où le recours au verbe fonctionnel bringen entraîne une modification de la structure syntactico-sémantique en tout point comparable au mécanisme général dégagé des définitions grammaticales discutées précédemment :

(12) Sonst musst Du wohl die MPEGs in ein anderes Format zur Bearbeitung bringen und danach wieder in MPEGs umwandeln.

'Sinon, tu dois sans doute changer le format des fichiers MPEG pour les traiter et, ensuite, les enregistrer à nouveau.'

Il n'est ainsi pas surprenant de retrouver parmi la liste des verbes fonctionnels productifs en allemand le couple stehen-stellen introduit dans la sous-partie précédente pour dégager la construction causative de base dans cette langue : stellen y fonctionne toujours comme variante causative de stehen, ainsi en (13) :

(13) a. Ein Stellplatz für Ihr Auto steht (Ihnen) zur Verfügung. 'Une place de parking est à votre disposition pour votre voiture.'

b. Wir stellen Ihnen einen sicheren Platz zur Verfügung und bewachen ihn rund um die Uhr.

'Nous mettons à votre disposition une place sécurisée que nous surveillons 24 heures sur 24.' 
Cet embryon de systématicité - repris dans la littérature sur la question sous la dénomination de sérialité (Reihenbildung) - a en partie plaidé, dans la tradition grammaticographique allemande, pour leur intégration dans le champ phraséologique. À ce premier argument s'est ajouté celui de l'affaiblissement du sens du verbe devenu fonctionnel, affaiblissement imprimant à l'ensemble de la structure un certain de degré de non-compositionnalité ${ }^{19}$. Celle-ci a alors débouché sur un traitement monolithique de ces structures envisagées comme des touts figés et en partie opaques. En témoignent, en particulier, les critères définitoires le plus souvent binaires établis par G. Helbig (1979) pour leur identification. Le corollaire de cette approche est toutefois, par delà les critères en question, d'avoir gommé la différence entre les deux types de verbes fonctionnels - aspectuels et causatifs - et, partant, entre les structures syntactico-sémantiques auxquelles ils aboutissent. Ainsi, le verbe fonctionnel causatif opère sur une prédication sousjacente contenant l'autre verbe fonctionnel non causatif tandis que les verbes fonctionnels aspectuels ne font « que » sur-imprimer une variante aspectuelle au prédicat exprimé par le $\mathrm{N}$ prédicatif sans modifier la structure argumentale de l'énoncé ${ }^{20}$. L'exemple (13b) repose en fait sur la structure de base suivante :

Wir CAUS ein sicherer Platz steht Ihnen zur Verfügung.

On voit donc apparaître là un intéressant point d'ancrage pour répondre aux objectifs définis en introduction: si la perception de ces structures comme des « touts monolithiques » les a intégrées nolens volens au domaine phraséologique, la possibilité de les analyser - malgré tout - en une opération syntacticosémantique comme l'opération consistant à transformer un énoncé non causatif en énoncé causatif invite à dépasser cette perspective et à les envisager sous un autre angle, celui d'une correspondance régulière forme-sens qui trouve, selon nous, dans les grammaires de construction un cadre d'analyse privilégié.

\section{LES CONSTRUCTIONS CAUSATIVES AVEC MOUVEMENT EN ALLEMAND}

\subsection{Vers un continuum de la causativité ?}

Si les deux types d'expression de la causativité examinés jusqu'ici peuvent être considérés comme plus ou moins prototypiques, essentiellement compte tenu de leur caractère semi-systématique, la question des structures sous-jacentes aux énoncés (3) et (4) commentés en introduction ou à l'énoncé (14) infra reste

19. Gries (2008: 6) discute ce critère en distinguant entre « unité sémantique » de la structure et noncompositionnalité du sens, seul le premier critère étant à ses yeux nécessaire. C'est là un point de jonction particulièrement pertinent avec les grammaires de construction (cf. § 4.2).

20. Ce que résume très bien Vivès (1993 : 14) même si son cadre théorique de départ s'inscrit pleinement dans la tradition française : «Un opérateur de causativité est un prédicat qui, lorsqu'il prend une phrase à verbe support comme deuxième argument, a comme effet syntaxique d'effacer le verbe support de cette phrase, d'en réajuster l'agencement et d'y ajouter un argument, son propre sujet. » 
entière : dans quelle mesure un verbe intransitif désignant la production d'un son comme klingeln ('sonner') peut-il être utilisé « transitivement » et avec un complément ablatif ?

(14) Sie hat ihren Freund aus dem Bett geklingelt.

'Elle a sorti son ami du lit en sonnant à la porte.'

Cet emploi du verbe klingeln ne correspond en effet ni à ses sens lexicographiés puisqu'il signifie ici réveiller, plutôt que sonner et, surtout, il est actualisé dans une configuration valencielle non prévue en dehors de ce type d'énoncé bien particulier. Un dictionnaire pour apprenants d'allemand langue étrangère comme G. Kempcke et al. (2000) répertorie ainsi les constructions suivantes :

jmd. klingelt $/ q q^{\prime}$ un sonne $\rightarrow$ eine Klingel betätigen/activer une sonnette etw., bes. es klingelt/qq.ch./ça sonne

bei jm. klingelt es/ça fait tilt pour qq'un

Bien qu'une construction « idiomatique », au sens athéorique du terme, soit prévue dans l'article (la dernière), aucune place n'est fait à la variante " transitive » et causative rencontrée en (14). Si la situation est légèrement différente dans le dictionnaire valenciel $\mathrm{Valbu}^{21}$, un examen précis des programmes argumentaux proposés montre que, même à ce niveau de description, la tentation est grande de recourir à l'explication phraséologique :

Remarques générales : (i) klingeln est aussi utilisé dans l'expression jemanden aus dem Bett/Schlaf klingeln ('sonner quelqu'un hors du lit/hors du sommeil') dans le sens de 'activer la sonnette ou laisser sonner le téléphone aussi longtemps que nécessaire pour que la personne se lève, se réveille et ouvre la porte ou réponde au téléphone' ; (ii) klingeln est utilisé occasionnellement avec un complément directif au sens de 'sonner aussi longtemps que nécessaire pour que quelqu'un se rende quelque part'. ${ }^{22}$

Ces emplois semblent y être considérés comme occasionnels ou périphériques : les définitions et paraphrases proposées insistent, d'une part, sur certaines restrictions (p. ex. pour ce qui est des groupes prépositionnels possibles) et posent, implicitement, la question de la part de sens attribuable au verbe et la part attribuable au groupe prépositionnel : si klingeln prend ici précisément ce sens dans cette « construction » particulière, peut-on encore en faire une valence du verbe en tant que tel ? C'est dans ce contexte qu'il faut comprendre le recours aux dimensions du figement et de l'idiomaticité ; ce recours est toutefois légèrement différent de celui qui a présidé au traitement phraséologique des locutions à verbe support examinées dans la section précédente dans la mesure où c'étaient essentiellement les restrictions morphosyntaxiques qui avaient conduit vers l'intégration au domaine de la phraséologie tandis que c'est là sur l'appariement entre forme et sens que l'analyse achoppe.

21. Consultable en ligne à l'adresse : http://www.ids-mannheim.de/gra/valbu.html (consulté le 2.3.2012).

22. Nous traduisons directement l'article allemand. 
Ces apories explicatives sont précisément celles que dénonce A. Goldberg (1995) dans sa discussion des approches qu'elle qualifie de lexicales, à savoir celles qui calculent le sens à partir du verbe et de sa complémentation :

The syntactic complement configuration of a clause is taken to be uniquely predictable from the semantic representation of the matrix verb. The mapping from semantic representations to particular complement configurations is performed via universal, or near universal, linking rules. Different syntactic complement configurations therefore reflect differences in the semantic representations of the main verb. (Goldberg, 1995 : 8)

Une construction comme celle rencontrée dans l'exemple (14) peut être comparée à celles, fréquentes en anglais, qui servent à exprimer un déplacement d'une entité, orienté par rapport à un repère, causé par un tiers et comportant un élément désignant les modalités de ce déplacement, comme dans l'exemple (15) utilisé par A. Goldberg (1995 : 173) :

(15) They laughed the poor guy into his car/out of the auditorium/off the stage. Ces structures, jusqu'ici peu étudiées en détails pour l'allemand, et qui ont des points communs avec les constructions résultatives (cf. Boas 2003 ; Müller 2002), sont certes moins productives, mais elles n'en sont pas moins intéressantes, justement compte tenu des limites théoriques qu'elles posent.

L'hypothèse défendue ici est qu'elles trouvent leur place le long d'un continuum composé de cinq degrés allant du plus grammaticalisé/figé au plus «idiomatique » :

- degré 1 : variante causative d'un verbe non causatif Er hat den Baum gefällt.

'Il a abattu l'arbre.'

- degré 2 : locution à verbe fonctionnel avec verbe causatif Wir stellen Ihnen einen sicheren Platz zur Verfügung.

'Nous mettons une place sûre à votre disposition.'

- degré 3 : verbe transitif encodant un déplacement associé à un complément directif

Er hat das Geld auf den Tisch gelegt.

'Il a posé l'argent sur la table.'

- degré 4.1 : verbe pouvant être transitif ou intransitif, employé transitivement encodant un déplacement associé à un complément directif

Er hat das Auto in den Graben gefahren.

'Il a mis la voiture dans le fossé.'

- degré $4.2^{23}$ : verbe intransitif employé de façon réfléchie encodant un déplacement associé à un complément directif

23. Ce cas de figure, qui présente toujours un pronom réfléchi en surface, est un cas limite que nous conservons ici par souci de complétude : on a, en effet, affaire deux fois à la même entité, une fois comme agent, une fois comme patient. 
Sie hat sich in die Herzen der Gäste getanzt.

'Elle a gagné le cœur des invités en dansant.'

- degré 4.3 : verbe transitif n'encodant pas de déplacement mais associé à un complément directif

Er hat das Kind in den Schlaf gewiegt.

'Il a endormi l'enfant en le berçant.'

- degré 5 : verbe intransitif employé transitivement n'encodant pas de déplacement mais associé à un complément directif

Sie hat ihren Freund aus dem Bett geklingelt.

'Elle a sorti son ami du lit en sonnant.'

Ce continuum est proche du classement des verbes résultatifs présenté par B. Levin et M. Rappaport Hovav (1995 : 36-37), mais il se limite ici aux seuls cas de « causativité avec mouvement».

\subsection{Discussion}

Parmi les formes figées, «formules » (idiomatiques ou non), dont l'importance a été soulignée dans les travaux sur le fonctionnement de la mémoire et sur l'apprentissage de la langue pour l'exécution des tâches communicatives (cf. Wray 1999, 2002 ; Wray \& Perkins 2000), les « constructions » n'ont émergé que tardivement. Il est aujourd'hui établi que, au-delà de la variation concernant son remplissage lexical, la structure est stockée en mémoire et reconnue, au même titre que d'autres formules lexicalement plus stables. La reconnaissance d'une construction comme " phrasème » est par ailleurs étroitement liée à la part de variation du remplissage lexical et donc à son degré de stabilité lexicale, et cette question divise les auteurs (Gries (2008) ou encore Dobrovol'skij (2011) qui défend l'idée selon laquelle ce remplissage doit être fixe).

Les constructions répertoriées dans le continuum supra, qui restent « classiques » avec des verbes transitifs exprimant un déplacement orienté (degré 3, du type Er legte das Geld auf den Tisch), deviennent particulièrement intéressantes dans les cas où le syntagme n'est plus explicable à partir du seul verbe, du moins à partir de sa syntaxe (degrés 4-5). Ces constructions utilisent un verbe qui, dans son emploi « standard », a au moins l'une des caractéristiques suivantes :

- il a un objet relevant d'une autre classe sémantique ;

- il n'est pas transitif ;

- il n'exprime pas un déplacement.

Nous rejoignons ici A. Goldberg (1995 : 152-153) qui déconnecte l'expression du mouvement du sémantisme du verbe pour l'attribuer à la construction ${ }^{24}$ et tenterons de déterminer dans ce qui suit quels facteurs sémantiques semblent

24. Voir Goldberg (1995 : 153) : "Many verbs do not necessarily code motion independently of this construction." 
faciliter l'entrée de certains verbes dans un complexe syntaxique à valeur causative.

Recourir à la notion de construction pour expliquer le fonctionnement syntaxique et sémantique du syntagme dans son ensemble nous paraît être une solution pertinente ici, et nous placerons l'interaction entre morphologie, syntaxe et sémantique au centre de notre problématique. C'est de fait cette interaction qui favorise l'émergence d'une unité interprétable en tant que telle, et ce, malgré les " déviances » syntaxiques et/ou sémantiques subies. Les approches mémorielles ont montré que les locuteurs recourent alors à une interprétation holistique pour chaque énoncé particulier - sur la base de la reconnaissance de la « construction » (Tomasello 2003 ; Wray \& Perkins 2000).

Cette notion est pertinente à différents niveaux :

- sur le plan syntaxique, elle permet de considérer le verbe ET son environnement comme une unité dont les éléments ne dépendent pas du seul verbe ;

- sur le plan sémantique, elle permet de considérer une répartition des rôles propres à la construction (et ne dépendant donc pas nécessairement de la valence du verbe) ;

- sur le plan cognitif, elle permet de rendre compte de la saisie du « réel » à travers des structures préexistantes à la parole (et indépendantes de certaines contraintes distributionnelles liées aux "mots»), mais certainement aussi liées à l'activation de "cadres cognitifs " (frames) ${ }^{25}$; de ce point de vue, le verbe joue un rôle central en initiant la mise en place d'une « scène " au sens athéorique du terme, qui constitue ainsi le premier niveau pour l'interprétation de la construction. Cette perspective sera en particulier mise à profit pour les constructions à verbe intransitif en ce qu'elle permet de les saisir comme le dernier degré d'abstraction sur un continuum prenant son origine dans la grammaire des cas de $\mathrm{C}$. Fillmore et passant par la sémantique des scènes et des cadres.

Avant d'en venir à la description de l'interaction entre les aspects morphologique, syntaxique et sémantique des constructions causatives qui nous intéressent, il est nécessaire de s'arrêter un instant sur les deux composantes de la construction : le complément directif, élément central dans l'expression de la causativité, et le groupe nominal à l'accusatif.

25. C'est la voie suivie par Martin (2008) pour analyser les collocations. Même si notre objet d'étude est à la fois différent et nettement plus abstrait que les collocations autour des instruments de musique analysées par l'auteur, les apports méthodologiques qu'il relève - au niveau de la représentation mentale d'une scène et au niveau de la production langagière - valent aussi pour la problématique au centre de cet article. 


\subsection{Le complément directif et la question du cas}

\subsubsection{Rappel sur les prépositions}

En dehors des prépositions à valeur directive à cas fixe (origine : aus, traversée : durch, but : zu), l'allemand dispose d'autres prépositions dont la caractéristique est de régir soit le datif, soit l'accusatif pour distinguer entre relation locative et relation directive ${ }^{26}$. L'accusatif est employé, avec des verbes exprimant un déplacement, chaque fois qu'il s'agit d'indiquer la direction de ce déplacement par rapport à un repère (point d'aboutissement du mouvement). On retrouve ici les verbes « causatifs » mentionnés supra (cf. § 2 ) :

(16) Sie legt das Heft auf den Schreibtisch. 'Elle pose le cahier sur le bureau.'

(17) Sie setzt das Kind neben seine Mutter. 'Elle assied l'enfant à côté de sa mère.'

\subsubsection{Activité vs. Action orientée}

Hormis les verbes mentionnés ici et relevant de mini-systèmes d'opposition, l'emploi de ce type de complément directif est intéressant avec des verbes qui, sans ce complément, désignent une simple activité (schwimmen, dribbeln, tanzen) [(18a), (19a), (20a)] et qui, avec ce complément, désignent une action [(18b), (19b), (20b)] orientée et bornée (" accomplissement »). Il est pertinent pour la suite de notre analyse de remarquer dès à présent que, pour un verbe comme tanzen ('danser'), la directivité quitte facilement le domaine spatial (20b) :

(18) a. Sie schwimmt jeden Morgen eine Stunde. 'Elle nage une heure chaque matin.'

b. Sie schwammen bis zu den Felsen. 'Ils nagèrent jusqu' aux rochers.'

(19) a. Der berühmte Spieler hat nicht gern gedribbelt. 'Ce célèbre joueur n'aimait pas dribbler.'

b. Markus Beer dribbelte in den Strafraum [...]. (Rhein-Zeitung, 5.6.2000 ${ }^{27}$ ) 'Markus Beer a dribblé (et amené le ballon) jusque dans la surface de réparation.'

(20) a. Sie tanzten jeden Sonntagnachmittag zusammen. 'Ils dansaient ensemble chaque dimanche après-midi.'

b. Wer noch nicht ins neue Jahr getanzt ist, kann es am 25. Januar nachholen! (Hannoversche Allgemeine, 19.12.2008)

'Qui n'a pas encore franchi le cap de la nouvelle année en dansant, peut encore se rattraper le 25 janvier.'

Ces mêmes verbes ont, par ailleurs, fréquemment un emploi réfléchi avec un complément directif. Il ne s'agit certes pas de causativité au sens strict, du fait 
$\mathrm{qu}^{\prime}$ agent et patient ne sont pas distincts, mais d'un simple point de vue formel, le rapprochement des structures est intéressant ${ }^{28}$ :

(21) Toni, der kleine Wasserfloh, schwamm sich in die Welt.

'Toni, la petite puce d'eau, est arrivé au monde à la nage.'

(22) Aurora sang und tanzte sich in die Herzen der Fiestagäste.

'Par ses chants et sa danse, Aurora est entrée dans les cœurs des participants à la Fiesta.'

L'importance du marquage de la directivité dans le groupe prépositionnel montre qu'on atteint, aux niveaux suivants, la limite des analyses partant de la structure argumentale du seul verbe. En effet, si dans le cas d'un verbe comme tanzen ou dribbeln, qui désigne une activité, celle-ci reste toujours liée à un mouvement qui est lui-même susceptible d'induire un déplacement orienté/borné ${ }^{29}$; en revanche, pour d'autres verbes, qui désignent une activité qui n'est pas liée à un mouvement (singen/chanter) et/ou ne peut a priori pas aboutir à un déplacement orienté (wiegen/bercer), le problème de l'interprétation du complément directif se pose :

(23) Vergeblich versucht der Vater ihn in den Schlaf zu singen.

'En vain, le père essaie de chanter pour l'endormir.' 30

(24) Es sollten die einzigen zwei Worte an diesem Nachmittag in Deutsch sein, der Sprache seiner Mutter, in der sie ihn einst im besetzten Frankreich manchmal leise in den Schlaf wiegte. (Die Weltwoche, 28.02.2007)

'Cela devait être, cet après-midi-là, les seuls mots en allemand, la langue de sa mère, dans laquelle elle le berçait jadis doucement, dans la France occupée, pour qu'il s'endorme.'

(25) Heute hat mich einer von e-plus um 7.00 Uhr aus dem Bett geklingelt.

'Aujourd'hui un type de e-plus a sonné à 7 heures et m'a tiré du lit.'

Dans de tels cas, en effet, mettre le complément directif en relation avec le seul verbe, comme dans les analyses traditionnelles discutées supra, conduirait à « bouleverser » à un tel point sa structure sémantique qu'il est préférable de recourir à un autre type de description. Si la directivité est imputable dans des cas tels que (18b)-(20b) au seul groupe prépositionnel marqué comme directif (préposition (18b) et/ou cas (19b)-(20b)), le jugement de cohérence de l'ensemble dans des cas tels que (15) et les exemples qui suivent dans l'illustration des différents degrés, c'est-à-dire leur interprétation comme constructions causatives, n'est pas liée à la seule présence - pourtant nécessaire ${ }^{31}$ - du groupe à l'accusatif (objet).

28. Voir note 23. Certains auteurs (van Valin 1990) considèrent le réfléchi comme servant à transformer l'activité en accomplissement et n'ayant pas de véritable fonction syntaxique.

29. On voit ici l'importance de la « scène » décrite par le verbe (cf. supra $§ 3.2$ ).

30. La traduction française fait bien ressortir ici la différence entre les deux langues : nous renvoyons aux notions de satellite-framing et verb-framing chez Talmy (2000) et à l'élargissement des catégories chez Croft et al. (2010) pour décrire des évènements complexes.

31. C'est ce que Carrier \& Randall (1992) appellent Direct Object Restriction. 
Afin d'examiner de plus près le fonctionnement du verbe et la manière dont il participe à l'interprétation causative de l'ensemble, il convient à présent de s'intéresser ici au statut de l'objet à l'accusatif.

\subsection{L'objet $1^{32}$}

Si l'on reprend ici les verbes évoqués supra pour les degrés 3 à 5 du continuum et que l'on examine le constituant à l'accusatif, on constate que l'on a affaire à :

- des verbes qui ont un emploi transitif (fahren, dribbeln);

- des verbes transitifs qui ne changent pas d'objet (wiegen);

- des verbes transitifs ou intransitifs employés avec un réfléchi (singen, tanzen);

- des verbes transitifs qui changent d'objet (singen, tanzen);

- des verbes intransitifs employés avec un accusatif (klingeln).

Les deux derniers cas sont particulièrement marqués et marquants (cf. supra (23)-(25)).

Le continuum de causativité présenté supra se double donc largement d'un autre continuum concernant cette fois la transitivité :

- des cas où le verbe garde son objet ;

- des cas (limites) où la place de l'accusatif est représentée/occupée par un pronom réfléchi ;

- des cas où le verbe change d'objet ;

- des cas, enfin, où le verbe, intransitif, entre dans une construction " transitive ».

Deux cas de figure se dessinent alors :

- les cas où le verbe conserve son objet, mais où celui-ci est soumis à un déplacement orienté ;

- les cas - les plus intéressants - où le verbe est accompagné d'un objet qui n'était pas «prévu » syntaxiquement ou sémantiquement, exemples (23)(25), mais qui ne pose pas de problème pour l'interprétation de son rôle de «patient » effectuant un mouvement orienté.

Notre première conclusion peut être formulée de la manière suivante : 1 'acceptabilité de l'énoncé et l'interprétation du procès décrit ne dépendent pas du seul verbe mais s'appuient à la fois sur une des propriétés sémantiques de celui-ci et sur notre connaissance/expérience du monde.

\footnotetext{
32. Nous appelons ici « Objet 1 » celui dont la relation au verbe est «première » : il s'agit dans la majorité des cas de l'objet à l'accusatif, mais il peut s'agir de l'objet au datif pour des verbes qui ne régissent que ce cas, tels que helfen, dont l'emploi dans des constructions causatives est d'ailleurs souvent cité en exemple : jmdm aus dem Wagen helfen ('aider qqn à sortir de la voiture'), jmdm in den Mantel helfen ('aider qqn à mettre son manteau'), jmdm aufs Pferd helfen ('aider qqn à monter sur son cheval'), etc.
} 
La dernière partie de notre étude nous permettra de redéfinir le rôle joué par le verbe et de donner une description sémantique de la construction avant de montrer qu'une telle approche nous ramène in fine vers le champ phraséologique.

\section{DESCRIPTION SÉMANTIQUE DE LA CONSTRUCTION}

Nous avons pu montrer à propos de cas tels que ceux illustrés par les exemples (23) à (25) que, d'un point de vue strictement valenciel, aussi bien le groupe « objet » à l'accusatif que le groupe à valeur directive ne dépendent pas nécessairement du verbe ; cette indépendance actantielle pose alors la vraie question de l'interprétation et de la description de ces constructions causatives. En effet, en dépit de l'indépendance sur le plan syntaxique, on constate que tout verbe ne tolère pas cette construction.

Sur le plan sémantique, mis à part le fait que les deux arguments (patient et lieu/but) sont à prendre en considération ensemble dans leur relation au prédicat, celui-ci doit pouvoir désigner une action " autorisant » le déplacement du patient. Dans tous les cas, c'est le recours à un cadre basé sur l'expérience qui permet l'interprétation directive (et causative). La mention du lieu (origine, passage ou but), d'une part, et celle du patient, d'autre part, sont à mettre en lien avec l'activité désignée par le verbe et - sur la base d'un cadre - s'effectue alors la ré-interprétation de l'activité comme action orientée. Cette nécessité d'un « cadre » explique que - malgré l'apparente liberté - tout verbe ne puisse donner lieu à une telle interprétation et que, comme nous allons le voir plus loin, certaines actions donnent lieu à des formes stéréotypées ${ }^{33}$. Si le passage au plan sémantique est donc nécessaire pour expliquer ce type de structures, cela ne signifie pas pour autant que le verbe s' « enrichit » de sens et que l'on doive voir ici un cas de polysémie. Deux raisons nous écartent de cette solution à la fois «facile », mais aussi complexifiante d'un point de vue lexical. D'une part, en effet, le lexème verbal continue, quel que soit son environnement, de désigner l'action ou l'activité qu'il désigne en dehors de son emploi dans des constructions causatives. D'autre part, les cas où le verbe est intransitif ou a un objet autre que celui utilisé dans la construction causative obligeraient à multiplier les « sens » en fonction des contextes en mettant systématiquement en relation l'objet et le lieu, ce qui deviendrait vite difficile en raison de la productivité de ces constructions. Si le verbe est assez « tolérant » pour accepter des emplois qui peuvent paraître contraires à son environnement " habituel ", c'est qu'il est intégré dans une structure qui condense des rôles sémantiques qui relèvent alors de cette structure, et non plus du seul verbe ${ }^{34}$.

33. C'est à la fois le degré de typicalité du lieu et celui de l'action qui y conduit qui autorisent le choix du verbe et son emploi comme verbe d'action. La productivité de ces constructions est liée à l'accessibilité de la « scène ».

34. Voir Rappaport Hovav \& Levin (1998 : 128), dont le propre cadre théorique n'est pas constructionnel mais « projectionniste » et qui décrivent l'intégration d'un verbe dans une construction de la manière suivante : 
La prise en compte de l'unité constructionnelle reflète les potentialités de la langue en les fondant sur la capacité des locuteurs à saisir les régularités de l'expérience, et elle permet en outre d'éviter le recours au figement et à la déviance, et donc à la multiplication des « sens ». En ouvrant la voie à un fonctionnement compositionnel, elle tient aussi compte des capacités mémorielles des locuteurs. La description sémantique de ce type de construction, en renonçant au recours au simple fait de figement particulier, replace le fait phraséologique à un autre niveau qu'il convient à présent de décrire.

\subsection{Métaphoricité}

La présence nécessaire d'un objet désignant le patient et d'un complément directif dans les constructions mentionnées supra montre une parenté avec des constructions similaires utilisant obligatoirement un pronom réfléchi qui occupe la place de l'accusatif, du type :

(26) Sie tanzte sich in die Herzen des Publikums.

'Elle a gagné le cœur du public en dansant.'

La fréquence (et la variété) de ces constructions " réfléchies » est grandement facilitée par la fixité de l'objet : le choix du verbe et du complément directif en «profitent » largement. Le fait le plus marquant est sans aucun doute la grande variation des "lieux», qui s'appuie sur un fonctionnement de type métaphorique : ainsi la fréquence du cœur (Herz) comme lieu visé par l'activité du sujet nous ramène du côté des nombreux phrasèmes utilisant ce formatif ${ }^{35}$.

Pour les constructions causatives avec un véritable objet patient, les cas de métaphoricité du lieu sont moins fréquents, toutefois on observe que c'est souvent un état qui représente le lieu (origine ou but). On trouve ainsi :

aus dem Schlaf, aus der Ruhe in den Schlaf, in die Einsamkeit

Dans tous ces cas, y compris ceux où il s'agit d'un véritable lieu, concret, i.e. ceux où le déplacement du patient se fait dans l'espace, le prédicat de la construction doit être décrit par l'ajout d'un trait de déplacement orienté. Les cas les plus intéressants restent, comme on l'a vu supra, ceux où la structure syntacticosémantique s'écarte nettement de celle du verbe et est propre à la construction.

Les faits de métaphoricité du lieu et la fréquence de certains lexèmes pouvant provoquer un certain figement nous ramènent du côté du phraséologique. Il reste à présent à voir en quoi le recours à cette dimension peut être pertinent pour la description des constructions causatives avec mouvement discutées ici. Une extension du domaine de la phraséologie, intégrant le niveau constructionnel, nous permet en effet de rendre compte du degré de figement et du fait

\footnotetext{
"The major constraint on integrating a verb into a construction is the 'compatibility' between the meaning of the verb and the meaning associated with the syntactic structures into which the verb is inserted."
}

35. Voir Hegedüs (1996) ; Staffeldt \& Ziem (2008). 
métaphorique en gardant la possibilité d'une description compositionnelle des formes idiomatiques au centre de cette étude.

\subsection{Retour vers le phraséologique ?}

À l'instar de l'argumentation développée à propos des locutions en sous par P. Lauwers (2010), notre positionnement en faveur d'une extension du champ phraséologique en direction des grammaires de construction doit tout d'abord être resitué historiquement et théoriquement. C'est la perspective adoptée par S. Gries (2008) qui compare la place de la réflexion phraséologique dans trois théories (grammaire générative, grammaire cognitive de Langacker et grammaires de construction de Goldberg) pour conclure que dans les deux dernières, le lien est pour ainsi dire consubstantiel. Un des articles toujours cité ${ }^{36}$ comme étant à l'origine de ce paradigme, celui de C. Fillmore, P. Kay et M. O'Connor (1988), s'intéresse précisément, à travers l'étude du connecteur polylexical anglais let alone, à l'inclusion des expressions idiomatiques dans un modèle holistique de description/explication de la langue. Mieux encore : les auteurs n'hésitent pas à partir de ces expressions, pourtant longtemps considérées comme étant à la marge - voire en dehors du système linguistique ${ }^{37}$ - pour revenir sur les traditionnelles oppositions entre, d'une part, lexique et grammaire et, d'autre part, figé/non figé. Aux trois catégories postulées dans la typologie de C. Fillmore, P. Kay et M. O'Connor (1988), W. Croft et D. Cruse (2004) en ajoutent $\mathrm{d}$ 'ailleurs une quatrième, celles des constructions totalement régulières validant ainsi l'hypothèse d'un continuum global constitué de quatre degrés :

- expressions lexicalement, sémantiquement et syntaxiquement irrégulières (ainsi celles comprenant des lexèmes n'apparaissant pas/plus en dehors de l'expression considérée, comme au fur et à mesure ou gang und gäbe) ;

- expressions lexicalement régulières, mais sémantiquement et syntaxiquement irrégulières (comme à vrai dire ou ganz zu schweigen) ;

- expressions lexicalement et syntaxiquement régulières, mais sémantiquement irrégulières (du type casser sa pipe ou den Teufel an die Wand malen) ;

- constructions lexicalement, syntaxiquement et sémantiquement régulières. ${ }^{38}$

$C^{\prime}$ est cette dernière classe qui se révèle particulièrement intéressante pour la problématique de ce numéro dans la mesure où elle semble ouvrir la porte à une véritable implosion du domaine phraséologique ${ }^{39}$. Nous souhaiterions toutefois montrer que ce risque est somme toute limité et que ce dernier degré a

36. Par exemple dans la mise en perspective historique de Legallois \& Gréa (2006).

37. Voir Gries (2008 : 10) : "It is probably fair to say that phraseology has generally played a rather limited role in the development of the various versions of generative grammar."

38. Cette synthèse s’inspire directement de la présentation de Legallois \& Gréa (2006 : 10).

39. Gries (2008 : 8) est d'ailleurs conscient de cette limite de sa définition reposant sur six critères. Il craint ainsi explicitement que le terme de «phraséologisme » lui-même devienne "a futile catch-all term devoid of empirical content." 
bien, ainsi que le soulignent D. Legallois et P. Gréa (2006), toute sa place sur le continuum :

Pour Fillmore et al., le seul moyen de rendre compte des connaissances phraséologiques du locuteur est la notion de Construction. Ainsi, une construction est une EI (= expression idiomatique) formelle (ou schématique au sens de Langacker) : les éléments de la construction sont lexicalement non saturés. Il y a donc apparemment une différence entre les EI substantielles et les EI formelles : les premières appartiennent au lexique, au répertoire ; les autres sont des entités plus ouvertes, plus accueillantes, et ne pouvant être prédites à partir de règles générales. Leurs propriétés syntaxiques, sémantiques, pragmatiques sont appariées à la forme (en tout cas pour les constructions les moins générales). Mais cette différence entre les deux types de EI est théorique ; empiriquement, les EI peuvent certes être saturées par des éléments lexicaux déterminés, mais ce cas reste marginal. Le raisonnement vaut également pour les EI formelles : elles peuvent être lexicalement ouvertes, mais également contenir quelques éléments déterminés. (Legallois \& Gréa, 2006 : 10)

Un des points forts d'une telle approche réside, par delà l'idée de continuum que nous avons instanciée ici pour le cas particulier de l'expression de la causativité, dans l'effacement, induit par ce modèle, de la frontière entre lexique et grammaire. Cette idée est elle aussi en très grande partie applicable au domaine traité dans cette étude. Les degrés 1 et 2 du continuum proposé supra relèveraient ainsi traditionnellement plutôt du lexique et les degrés 3 à 5 de la grammaire, même si la démonstration qui précède a montré que ces différents cas reposent tous sur une construction schématique sous-jacente commune dont les contraintes d'instanciation vont toutefois augmentant du degré 3 au degré 5. La différence essentielle entre ces degrés étant la nature, tant syntaxique (transitif ou non) que sémantique (induisant un mouvement/déplacement ou non), du verbe, c'est sans nulle doute à ce niveau que les notions de figement et d'idiomaticité peuvent être (ré-)introduites.

C'est par exemple le cas dans le Lexical Constructional Model de R. de Mendoza (cité par Luzondo Oyón 2007) qui postule un changement de niveau pour les représentations liées au verbe décrit comme un phénomène métaphorique du type AN EXPERIENTAL ACTION IS AN EFFECTUAL ACTION. Ce schème général, qui serait donc actualisé non pas au niveau du seul verbe - pour en faire un " sens » supplémentaire - mais bel et bien au niveau de la construction dans son ensemble rejoint en partie l'explication que nous avons avancée (§ 3.3.2) d'une activité générale devenant une action bornée affectant un patient. La compatibilité de ce schème n'étant pas donnée pour tous les verbes, les restrictions observées pour le degré 5 reposent donc bien, derrière la construction générale, sur une question de figuration qui réoriente ces structures nolens volens vers le lexique ; la créativité, si elle n'est pas exclue, restant finalement limitée.

La question qui se pose donc in fine pour justifier le traitement à la fois constructionnel et phraséologique de ces structures est bien celle de savoir s'il faut considérer le schéma global isolé dans cet article comme une "simple » structure argumentale lexicalement non saturée et susceptible de multiples 
réalisations ou bien comme une structure argumentale présentant certaines restrictions, justement analysables du point de vue phraséologique. Car c'est là que semble se jouer, par exemple pour D. Dobrovol'skij (2011 : 112-113), la répartition des rôles entre les deux approches. S. Gries (2008) suit la même voie en mettant comme condition préalable à la reconnaissance du statut de phraséologisme la présence d'au moins un élément lexical contraint, que ce soit un lexème nu ou une forme fléchie :

In sum, a phraseologism is defined as the co-occurrence of a form or a lemma of a lexical item and one or more additional linguistic elements of various kind which functions as one semantic unit in a clause or sentence and whose frequency of cooccurrence is larger than expected on the basis of chance. (Gries, $2008: 6$ )

Ce faisant, S. Gries exclut du domaine les constructions lexicalement sousspécifiées (op. cit. : 8).

À nos yeux, les degrés 4 et 5 du continuum justifient toutefois pleinement ce double traitement : si la construction en elle-même reste bien la construction générale abstraite à la base de tout le continuum, ses conditions de réalisation, voire son " mode d'emploi », conditionnent à un tel point la sélection du verbe que nous sommes bien en présence d'un phénomène de figement permettant de reformuler une des conclusions intermédiaires de D. Dobrovol'skij (2011) :

Plus la construction en question est abstraite et productive, plus elle est proche du pôle grammatical sur le continuum lexique-grammaire. Plus elle est 'phraséologique', c'est-à-dire plus elle est contrainte sémantiquement, plus la construction en question est proche du pôle lexical. (Dobrovol'skij, $2011: 113)^{40}$

Les résultats auxquels nous sommes donc parvenus au terme de cette étude demandent à être confirmés dans au moins deux directions : d'une part, dans une perspective diachronique - par exemple à l'instar du travail de T. Colleman et B. de Clerck (2011) sur les constructions ditransitives en anglais - afin de mesurer, sur le moyen et le long terme, le degré de productivité de chacun des degrés postulés et de constater, surtout pour les degrés 4 et 5, si une évolution est en cours ; d'autre part, dans une perspective acquisitionnelle afin de voir si les degrés du continuum et leur répartition entre les deux pôles grammaticaux et lexicaux correspondent à des phases d'apprentissage différentes.

\section{Références}

BoAs H. (2003), A Constructional Approach to Resultatives, Stanford: CLSI Publications.

BuRger H. (1998 [2010 $]$ ), Phraseologie. Eine Einführung am Beispiel des Deutschen, Berlin : Erich Schmidt.

\footnotetext{
40. La spécification concerne ainsi davantage le poids des contraintes sémantiques exercées par la construction sur le verbe que son degré de saturation lexicale. Cf. l'original de Dobrovol'skij (2011: 113) : « Je abstrakter und produktiver die jeweilige Konstruktion ist, desto näher befindet sie sich am grammatischen Pol auf der Lexikon-Grammatik-Achse. Je 'phraseologischer', d.h. je stärker lexikalisch spezifiziert sie ist, desto näher liegt die betreffende Konstruktion am lexikalischen Pol. »
} 
CARRIER J. \& Randall J. H. (1992), “The Argument Structure and Syntactic Structure of Resultatives", Linguistic Inquiry 23 (2), 173-234.

Colleman T. \& de Clerck B. (2011), "Constructional semantics on the move: On semantic specialization in the English double object construction", Cognitive Linguistics 22 (1), 183209.

CROFT W. \& CRUSE D. A. (2004), Cognitive Linguistics, Cambridge (UK): Cambridge University Press.

CROFT W. et al. (2010), "Revising Talmy's typological classification of complex events", in $\mathrm{H}$. Boas (ed.), Contrastive construction grammar, Amsterdam/Philadelphia: John Benjamins, 201-235.

DobrovoL'SKIJ D. (2011), "Phraseologie und Konstruktionsgrammatik ", in A. Lasch \& A. Ziem (éds), Konstruktionsgrammatik III. Aktuelle Fragen und Lösungsansätze, Tübingen : Stauffenburg, 111-130.

FEILKE H. (2007), "Syntaktische Aspekte der Phraseologie III : Construction Grammar und verwandte Ansätze ", in $\mathrm{H}$. Burger et al. (éds), Phraseologie : ein internationales Handbuch zeitgenössischer Forschung, Berlin/New York : de Gruyter, 63-76.

FILLMORE C. J., KAY P. \& O'CONNOR M. C. (1988), “Regularity and Idiomaticity in Grammatical Constructions: the Case of Let Alone", Language 64 (3), 501-538.

FLEISCHER W. (1982 [1997²]), Phraseologie der deutschen Gegenwartssprache, Leipzig : VEB Bibliographisches Institut.

GAUTIER L. (2012), Pour une approche linguistique intégrative des discours spécialisés, Document de synthèse méthodologique en vue de I'HDR, Paris : Université Paris-Sorbonne.

GautieR L. (sous presse), "Prädikatives N / N prédicatif : retour sur la fortune de deux notions dans les traditions française et allemande ", in L. Gautier \& M.-H. Viguier (éds), Les noms prédicatifs : regards contrastifs français-allemand, Frankfurt/Main : Peter Lang.

Goldberg A. E. (1995), Constructions. A Construction Grammar Approach to Argument Structure, Chicago: The University of Chicago Press.

Goldberg A. E. (2006), Constructions at Work. The Nature of Generalization in Language, Oxford: Oxford University Press.

Granger S. \& Meunier F. (eds) (2008), Phraseology. An Interdisciplinary Perspective, Amsterdam/Philadelphia: John Benjamins.

GRÉCIANO G. (1983), Signification et dénotation en allemand. La sémantique des expressions idiomatiques, Paris : Klincksieck.

Gries S. (2008), "Phraseology and linguistic theory: A brief survey", in S. Granger \& F. Meunier (eds), Phraseology: An Interdisciplinary Perspective, Amsterdam/Philadelphia: John Benjamins, 3-25.

Hegedüs C. (1996), Phraséologie contrastive: la saisie des cinquante formatifs les plus productifs en allemand et en français, Thèse de l'Université Strasbourg 2.

HELBIG G. (1979), "Probleme der Beschreibung von Funktionsverbgefügen im Deutschen ", Deutsch als Fremdsprache 16, 273-285.

KEMPCKE G. et al. (2000), Wörterbuch Deutsch als Fremdsprache, Berlin : de Gruyter.

LAUWERS P. (2010), "Les locutions en sous comme constructions ", Le Français moderne 78 (1), 3-27.

Legallois D. \& GréA P. (2006), "La Grammaire de Construction ", Cahier du CRISCO 21, 5-27.

LEVIN B. \& RAPPAPORT Hovav M. (1995), Unaccusativity. At the Syntax-Lexical Semantics Interface, Cambridge (MA): MIT Press. 
LEVIN B. \& RAPPAPORT Hovav M. (2001), “An Event structure account of English resultatives”, Language 77 (4), 766-797.

LuZONDO OYón A. (2007), "Semantic constraints on the caused-motion construction", Epos 23, 167-180.

MARTIN W. (2008), “A unified approach to semantic frames and collocational patterns", in S. Granger \& F. Meunier (eds), Phraseology: An interdisciplinary perspective, Amsterdam/Philadelphia: John Benjamins, 51-65.

MEJRI S. (1997), Le figement lexical. Descriptions linguistiques et structuration sémantique, Tunis : Publications de la Faculté des Lettres de la Manouba.

Müller S. (2002), Complex Predicates: Verbal Complexes, Resultative Constructions, and Particle Verbs in German, Stanford: CSLI Publications.

NeVEu F. (2004), Dictionnaire des sciences du langage, Paris : Armand Colin.

RAPPAPORT Hovav M. \& LeVIN B. (1998), “Building Verb Meanings”, in M. Butt \& W. Geuder (eds), The Projection of Arguments. Lexical and Compositional Factors, Stanford: CSLI Publications, 97-131.

Schmale G. (2013), "Qu'est-ce qui est préfabriqué dans la langue ? - Réflexions au sujet d'une définition élargie de la préformation langagière ", Langages 189, Paris : Larousse/Armand Colin (ce volume).

STAFFELDT S. \& ZiEM A. (2008), "Körper-Sprache : Zur Motiviertheit von Körperteilbezeichnungen in Phraseologismen ", Sprachwissenschaft 33 (4), 455-499.

TALMY L. (2000), Toward a cognitive semantics, vol. 2: Typology and process in concept structuring, Cambridge (MA): MIT Press.

Tomasello M. (2003), Constructing a Language: A Usage-Based Approach to Child Language Acquisition, Cambridge (MA): Harvard University Press.

Van Pottelberge J. (2001), Verbonominale Konstruktionen, Funktionsverbgefüge. Vom Sinn und Unsinn eines Forschungsgegenstandes, Heidelberg : Winter.

VAN Pottelberge J. (2007), " Funktionsverbgefüge und verwandte Erscheinungen ", in H. Burger et al. (eds), Phraseologie: Ein internationales Handbuch zeitgenössischer Forschung, Berlin/New York : de Gruyter, 436-444.

VAN VALIN R. D. (1990), “Semantic parameters of split intransitivity”, Language 66, 221-220.

VIVĖs R. (1993), "La prédication nominale et l'analyse par verbes supports ", L'Information grammaticale 59, 8-15.

Von Polenz P. (1963), Fuktionsverben im heutigen Deustch. Sprache in der rationalisierten Welt, Düsseldorf : Schwann.

Von Polenz P. (1985), Deutsche Satzsemantik. Grundbegriffe des Zwischen-den-Zeilen-Lesens, Berlin/New York : de Gruyter.

WRAY A. (1999), "Formulaic language in learners and native speakers", Language Teaching 32 (1), 213-231.

WraY A. (2002), Formulaic Language and the Lexicon, Cambridge: Cambridge University Press.

WraY A. (2008), Formulaic Language: Pushing the Boundaries, Oxford: Oxford University Press.

WRAY A. \& PERKINS M. R. (2000), “The functions of formulaic language: an integrated model”, Language \& Communication 20, 1-28.

ZWARST J. (2010), "A hierarchy of locations: evidence from the encoding of direction in adpositions and cases", Linguistics 48 (5), 983-1009. 